\title{
AVALIAÇÃO DE UM CIRCUITO ELETRÔNICO PARA MEDIÇÃO DA CONDUTIVIDADE ELÉTRICA DE SOLUÇÕES NUTRITIVAS
}

\section{ANTONIO J. STEIDLE NETO ${ }^{1}$, SÉRGIO ZOLNIER ${ }^{2}$, WALDIR A. MAROUELLI ${ }^{3}$, OSMAR A. CARRIJO ${ }^{4}$, HERMINIA E. P. MARTINEZ ${ }^{5}$}

\begin{abstract}
RESUMO: Nos sistemas de cultivo hidropônicos ou que utilizam a fertirrigação, a quantidade total de sais dissolvidos na solução nutritiva tem grande impacto na produtividade da cultura. A concentração adequada ao crescimento e desenvolvimento da cultura sofre variações marcantes ao longo do seu ciclo, podendo ser avaliada indiretamente por meio do monitoramento da condutividade elétrica da solução nutritiva. $\mathrm{O}$ objetivo deste trabalho foi avaliar um circuito eletrônico de baixo custo para a medição da condutividade elétrica, cujo princípio se baseia na medida de freqüência. $\mathrm{O}$ circuito eletrônico foi conectado a um computador via porta paralela, sendo calibrado utilizando-se de 16 soluções de $\mathrm{KCl}$. A temperatura das soluções foi mantida nos níveis desejados por meio de um equipamento de controle da temperatura. Análises de regressão foram utilizadas para obter relações entre frequiência e resistência, visando a medir a condutividade elétrica e a temperatura da solução com base na freqüência. Os coeficientes de determinação obtidos demonstraram excelentes correlações $\left(\mathrm{R}^{2}\right.$ $>$ 0,997). Com base nos resultados, pôde-se concluir que o circuito proposto é capaz de medir a condutividade elétrica de soluções na faixa de 0,10 a $10,15 \mathrm{dS} \mathrm{m}^{-1}$ com grande exatidão, compensando automaticamente as variações instantâneas de temperatura.
\end{abstract}

PALAVRAS-CHAVE: condutivímetros, hidroponia, fertirrigação.

\section{EVALUATION OF AN ELECTRONIC CIRCUIT TO MEASURE ELECTRICAL CONDUCTIVITY OF NUTRIENT SOLUTION}

\begin{abstract}
In hydroponic systems or those that use the fertigation, the total amount of soluble salts in the nutrient solution has a significant impact on crop productivity. An adequate fertilizer concentration can be modified in response to stages of plant development through indirect monitoring of electrical conductivity of nutrient solution. The objective of this work was to evaluate a low cost electronic circuit to measure the electrical conductivity based on frequency measurements. The electronic circuit was connected to a computer using the parallel interface and calibrated with $16 \mathrm{KCl}$ solutions. The solutions temperature was maintained at desired levels with a controlled temperature equipment. Regression analysis was used to obtain relationships between frequency and resistance in order to measure the electrical conductivity and the solution temperature based on measured data of frequency. The coefficients of determination obtained from this study showed excellent correlations $\left(\mathrm{R}^{2}>0.997\right)$. The results demonstrated that the proposed circuit is capable of measuring the electrical conductivity of the solution from 0.10 to $10.15 \mathrm{dS} \mathrm{m}^{-1}$ with considerable accuracy, compensating automatically instantaneous temperature variations.
\end{abstract}

KEYWORDS: conductivity meters, hydroponic, fertigation.

\footnotetext{
${ }^{1}$ Eng $^{\text {o }}$ Agrícola, Doutorando em Agronomia, Departamento de Engenharia Agrícola, Universidade Federal de Viçosa, Viçosa - MG, Fone: (0XX31) 3899.1891, steidle@ vicosa.ufv.br

${ }^{2}$ Eng ${ }^{\circ}$ Agrícola, Prof. Doutor, Departamento de Engenharia Agrícola, UFV, Viçosa - MG, zolnier@ufv.br

${ }^{3}$ Eng $^{\circ}$ Agrícola, Pesquisador, Embrapa Hortaliças, Brasília - DF, waldir@ cnph.embrapa.br

${ }^{4}$ Eng ${ }^{\mathrm{O}}$ Agrônomo, Pesquisador, Embrapa Hortaliças, Brasília - DF, carrijo@ cnph.embrapa.br

${ }^{5}$ Eng ${ }^{\mathrm{a}}$ Agrônoma, Profa. Doutora, Departamento de Fitotecnia, UFV, Viçosa - MG, herminia@ufv.br

Recebido pelo Conselho Editorial em: 24-11-2004

Aprovado pelo Conselho Editorial em: 11-7-2005
} 


\section{INTRODUÇÃO}

A condutividade elétrica de uma substância é definida como a capacidade dessa em conduzir corrente elétrica. O princípio no qual a maioria dos equipamentos de medida da condutividade elétrica se baseia, consiste na medida de tensão quando uma corrente alternada é aplicada em dois eletrodos de uma célula de condutividade elétrica imersa em uma solução (RADIOMETER ANALYTICAL, 2004).

Geralmente, o termo resistividade é associado à condutividade elétrica, já que, com a imersão de dois eletrodos em solução, existirá maior ou menor resistência à passagem de corrente elétrica, dependendo da concentração da solução. A relação entre condutividade elétrica e resistividade é inversamente proporcional.

A condutividade elétrica é dependente da temperatura de tal forma que, se essa aumenta, a resistência da solução à passagem de corrente diminui, resultando em acréscimo na condutividade. Para fins de padronização, ao fazer referência sobre a condutividade elétrica de uma solução, deve-se sempre reportar à temperatura de $25^{\circ} \mathrm{C}$.

Em sistemas hidropônicos, a composição da solução nutritiva varia de acordo com o crescimento das plantas. Essa variação, induzida pelo crescimento, causa decréscimo nas quantidades de sais disponíveis para as raízes (MARTINEZ, 1999). Por esse motivo, a manutenção de meio favorável ao crescimento e desenvolvimento das plantas requer o monitoramento contínuo da solução nutritiva. Nesse sentido, medidas da condutividade elétrica são extremamente úteis, pois possibilitam o controle da concentração total de sais dissolvidos na solução nutritiva.

FURLANI et al. (1999) recomendam que, para hortaliças de folhas, como agrião, alface, almeirão, cebolinha, chicória, rúcula e salsa, a condutividade elétrica da solução nutritiva deve ser mantida entre $1,0 \mathrm{e} 1,2 \mathrm{dS} \mathrm{m}{ }^{-1}$ durante a fase de mudas e entre $1,4 \mathrm{e} 1,6 \mathrm{dS} \mathrm{m}{ }^{-1}$ na fase de produção. No caso das hortaliças de frutos, a condutividade elétrica recomendada durante a fase de muda é a mesma das hortaliças de folhas, porém, na fase de produção, as exigências nutricionais de culturas, como melão, pepino, pimentão e tomate, são maiores, sendo sugeridas condutividades para a solução nutritiva entre 2,0 e $4,0 \mathrm{dS} \mathrm{m}^{-1}$.

No Brasil, existe grande carência de literatura especializada em automação de sistemas de cultivo hidropônicos que apresente maneiras eficientes de monitorar e controlar a condutividade elétrica de soluções nutritivas.

A maioria dos equipamentos nacionais de medida da condutividade elétrica disponíveis comercialmente permite ao usuário apenas a obtenção de valores instantâneos de condutividade, tendo algumas exceções, funções de transferência para computador e/ou memória para o armazenamento de poucos dados. Assim sendo, não possibilitam o acompanhamento da variação da condutividade elétrica de uma solução por longos períodos de tempo, inviabilizando a implementação de sistema de controle da condutividade. Adicionalmente, esses equipamentos representam uma parcela expressiva dos custos de um sistema de produção hidropônico.

Dessa forma, é evidente a necessidade de um equipamento de baixo custo para medir a condutividade elétrica de soluções, o qual possibilite o monitoramento, em tempo real e de maneira contínua, e que seja facilmente adaptado a um sistema de controle, com vistas a otimizar o processo produtivo de vegetais.

O presente trabalho teve por objetivo avaliar um circuito eletrônico de baixo custo para a medição da condutividade elétrica, cujo princípio se baseia na medida de frequiência e não na diferença de potencial elétrico entre os eletrodos de uma célula de condutividade. O circuito eletrônico e as equações de calibração apresentadas neste trabalho foram patenteados junto ao INPI (Instituto Nacional da Propriedade Industrial) sob número PI0502492-7. 


\section{MATERIAL E MÉTODOS}

O circuito para medição da condutividade elétrica, constituído por seis componentes eletrônicos simples, foi montado em matriz de contato (Figura 1). O custo do circuito, considerando todos os componentes, foi de $\mathrm{R} \$ 4,00$, aproximadamente. Quando alimentado por uma fonte de corrente contínua, obteve-se, nos terminais de saída do circuito, um sinal alternado, cuja freqüência de oscilação medida foi relacionada ao valor verdadeiro de um dos resistores do circuito. Dessa maneira, fez-se um estudo preliminar a fim de estabelecer uma relação entre freqüência e resistência, pesquisando como a variação do valor do resistor afeta a freqüência medida nos terminais de saída do circuito. Essa relação foi estabelecida mediante a substituição do resistor de valor fixo do circuito por outros de valores ôhmicos conhecidos e medindo-se, individualmente, a freqüência associada. A variação do valor ôhmico do resistor simulou maior ou menor resistência à passagem de corrente elétrica em uma solução nutritiva.

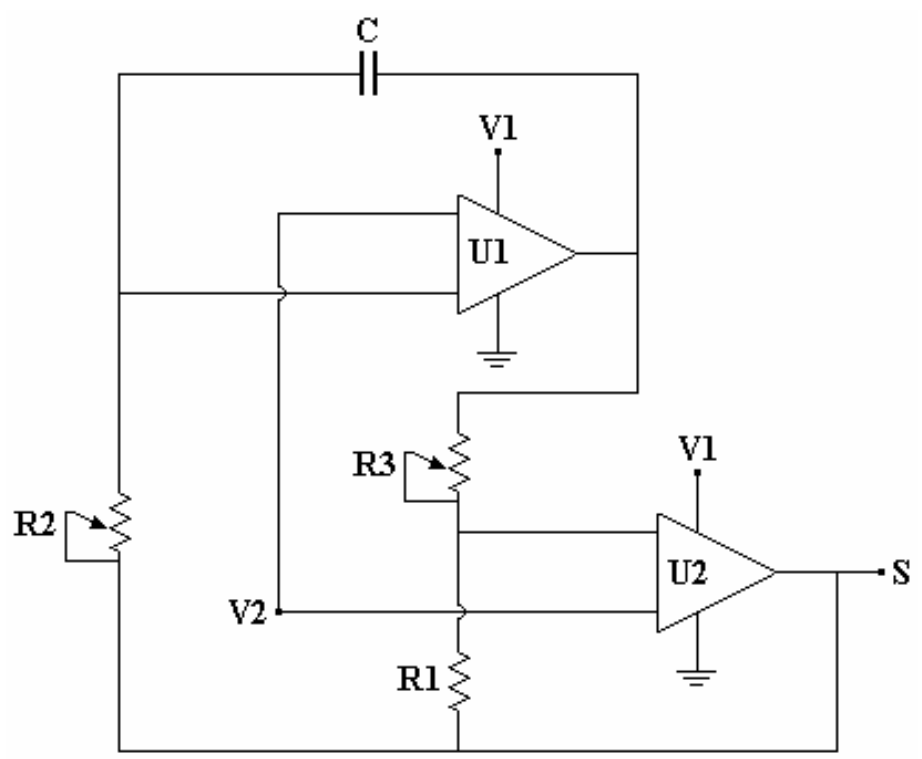

FIGURA 1. Diagrama eletrônico do circuito de medição da condutividade elétrica.

Inicialmente, determinaram-se os valores ôhmicos verdadeiros dos resistores a serem utilizados para a obtenção da referida relação. Dessa forma, foram adquiridos resistores da série comercial com valores ôhmicos nominais variando de 100 a $10.000 \Omega$, totalizando 29 pontos. O equipamento empregado na medição do valor ôhmico verdadeiro de cada resistor foi a Ponte de Thomson (modelo YTW, fabricada pela Ganz).

A conexão entre o circuito de medição da condutividade elétrica e o computador foi feita via porta paralela. Apesar de essa interface de comunicação ter sido desenvolvida para conectar periféricos, como impressoras ou scanners a computadores, ela pode ser utilizada para enviar ou receber informações de outros equipamentos (AXELSON, 1996). O conector DB25, responsável por essa interface, é composto por 25 pinos, dentre os quais cinco podem ser utilizados para receber dados e doze para enviar dados com base nos níveis lógicos baixo $(0,0$ a 0,8 Vcc) e alto $(2,0$ a 5,0 Vcc), padrão TTL (Transistor Transistor Logic). Nesse circuito, como era desejado medir a freqüência do sinal nos terminais de saída, foi necessário apenas um dos cinco pinos disponíveis para a recepção de dados.

Um programa computacional foi desenvolvido para gerenciar a aquisição de dados de freqüência, sendo implementado em linguagem $\mathrm{C}++$, utilizando-se da plataforma de programação $\mathrm{C}++$ Builder versão 5. Esse programa foi nomeado Hidro-CE (programa de aquisição de dados de freqüência para o cálculo da condutividade elétrica de soluções nutritivas aplicadas ao cultivo 
hidropônico). A cada minuto, o programa calculou a razão entre o número de pulsos e o número de segundos, gerando valor de freqüência em hertz.

A relação entre freqüência e resistência foi obtida por meio de modelo de regressão com três parâmetros [eq.(1)]. Visando ao melhor ajuste do modelo aos dados experimentais medidos, foram geradas duas equações abrangendo as faixas de condutividade elétrica de 0,1 a $2,15 \mathrm{dS} \mathrm{m}^{-1}$ e 2,15 a $10,15 \mathrm{dS} \mathrm{m}^{-1}$.

$$
F=\frac{a\left( \pm S E_{a}\right)+b\left( \pm S E_{b}\right) R}{1+c\left( \pm S E_{c}\right) R}
$$

em que,

F - freqüência, $\mathrm{Hz}$;

a, b, c - parâmetros da equação de regressão;

$\mathrm{R}$ - resistência, $\Omega$, e

SEi - desvio-padrão associado ao parâmetro i, i = a, b, c.

As equações de regressão geradas foram implementadas no Hidro-CE, possibilitando, por conseguinte, obter valores de resistência calculados a partir dos dados de freqüência medidos. Cada valor de resistência foi invertido e multiplicado por um fator igual a 1.000, resultando no valor correspondente de condutividade elétrica em dS m $\mathrm{m}^{-1}$.

Uma célula de condutividade (modelo EW-19500-20, fabricada pela Cole-Parmer) foi utilizada em substituição ao resistor no circuito. Essa célula apresenta corpo de vidro e dois eletrodos revestidos com platina. No interior do corpo da célula, um termistor de $10 \mathrm{k} \Omega$, com coeficiente de temperatura negativo (NTC), permite medir a temperatura do meio onde a célula está inserida. Na extremidade do cabo da célula de condutividade, além do par de fios correspondente aos eletrodos (conectado ao circuito para medição da condutividade elétrica), existe outro par referente ao sensor de temperatura. Esse segundo par de fios foi conectado a um circuito semelhante ao de medição da condutividade elétrica. Por meio da temperatura medida com esse circuito, foi possível realizar a compensação da condutividade elétrica da solução em relação à referência $\left(25^{\circ} \mathrm{C}\right)$.

Estabelecida a relação entre freqüência e condutividade elétrica, bastava calibrar o circuito eletrônico de medição da condutividade. Para esse procedimento, foram preparadas 16 soluçõespadrão de cloreto de potássio $(\mathrm{KCl})$ com as seguintes concentrações: 0,$001 ; 0,002 ; 0,003 ; 0,004$; 0,005; 0,006; 0,007; 0,008; 0,009; 0,01;0,02;0,03;0,04; 0,05; 0,06;0,07 N. As soluções-padrão foram obtidas a partir do preparo da solução base de $\mathrm{KCl}$ com concentração de $0,01 \mathrm{~N}$, a qual possui condutividade elétrica de $1,4118 \mathrm{dS} \mathrm{m} \mathrm{m}^{-1}$ à temperatura de $25^{\circ} \mathrm{C}$ (GARTLEY, 1995). Para cada concentração, foi utilizado um volume de $150 \mathrm{~mL}$, acondicionado em recipiente plástico hermeticamente fechado, a fim de evitar a evaporação e a conseqüente alteração da concentração.

Para que a calibração do circuito de condutividade fosse conduzida de maneira adequada, foi necessário controlar a temperatura das soluções-padrão. Dessa forma, empregou-se um equipamento de banho termostatizado com circulação forçada de água (modelo Te-184, faixa de operação de -10 a $100{ }^{\circ} \mathrm{C}$, fabricado pela Tecnal) que possibilita o controle da temperatura da água do banho com precisão de $\pm 0,1^{\circ} \mathrm{C}$. Entretanto, em razão de o campo eletromagnético gerado pelo equipamento provocar alterações significativas nos valores de freqüência do circuito, na medição de condutividade, tornou-se obrigatório o uso de uma caixa de isopor para o acondicionamento térmico das soluçõespadrão, mantida afastada aproximadamente $1,5 \mathrm{~m}$ do equipamento. Por meio de mangueiras de borracha, a água com temperatura controlada foi conduzida do equipamento de banho à caixa de isopor e vice-versa, fazendo com que a temperatura da água contida na caixa de isopor fosse mantida conforme desejado. 
O monitoramento da temperatura da água na caixa de isopor foi realizado por meio de termômetro-padrão de mercúrio em vidro, com escala nominal graduada de 0 a $50{ }^{\circ} \mathrm{C}$ e certificado de calibração do NIST (National Institute of Standards and Technology). O circuito de medição da condutividade foi calibrado considerando-se as temperaturas de $15 ; 25$ e $35^{\circ} \mathrm{C}$, pois as temperaturas normalmente encontradas nos sistemas hidropônicos existentes no Brasil estão entre 15 e $35^{\circ} \mathrm{C}$. No presente trabalho, utilizaram-se três células de condutividade elétrica, cujas características foram descritas anteriormente.

Para medir a temperatura da solução por meio do termistor de $10 \mathrm{k} \Omega$, conectado ao segundo circuito, foi necessário determinar equações de regressão que relacionassem freqüência com resistência e resistência com temperatura. A primeira relação foi estabelecida de maneira idêntica à descrita para o circuito de condutividade, usando-se os resistores de valores ôhmicos verdadeiros medidos por meio da Ponte de Thomson e o modelo de regressão com três parâmetros [eq.(1)].

Similarmente à medição de condutividade, o circuito de medição da temperatura foi conectado ao computador via porta paralela, mas por meio de outro pino disponível para a recepção de dados.

A relação entre resistência e temperatura foi determinada por meio de calibração, comparando-se as medidas de resistividade elétrica do termistor com os valores correspondentes de temperatura na faixa de 10 a $40^{\circ} \mathrm{C}$, obtidos com o termômetro-padrão de mercúrio em vidro. Nessa calibração, empregou-se um recipiente cilíndrico termicamente isolado, sendo feitos dois orifícios circulares na tampa desse para a inserção da célula de condutividade e do termômetro-padrão.

O modelo de regressão utilizado para estabelecer a relação entre resistência e temperatura foi o descrito por DeFELICE (1998) para sensores de temperatura do tipo termistor [eq.(2)]. A equação obtida foi posteriormente implementada no Hidro-CE.

$$
\mathrm{R}=\mathrm{R}\left(\mathrm{T}_{\text {ref }}\right) \exp \left\{\beta\left( \pm \mathrm{SE}_{\beta}\right)\left\lfloor(\mathrm{T}+273,16)^{-1}-\left(\mathrm{T}_{\text {ref }}+273,16\right)^{-1}\right]\right\}
$$

em que,

$\mathrm{R}\left(\mathrm{T}_{\mathrm{ref}}\right)$ - resistência à temperatura de referência, $\Omega$;

$\beta$ - parâmetro da equação de regressão, $K$;

SE $\beta$ - desvio-padrão associado ao parâmetro $\beta$;

$\mathrm{T}$ - temperatura, ${ }^{\circ} \mathrm{C}$, e

$\mathrm{T}_{\text {ref }}$ - temperatura de referência, $25^{\circ} \mathrm{C}$.

Visando à correção da condutividade elétrica de soluções com temperaturas diferentes do valor de referência $\left(25^{\circ} \mathrm{C}\right)$, realizou-se regressão múltipla, com base na equação linear apresentada por RADIOMETER ANALYTICAL (2004), a qual envolve um coeficiente de temperatura $(\alpha)$ dependente da solução [eq.(3)].

em que,

$$
\mathrm{CE}=\mathrm{CE}\left(\mathrm{T}_{\text {ref }}\right)+0,01\left[\mathrm{CE}\left(\mathrm{T}_{\text {ref }}\right) \alpha\left( \pm \mathrm{SE}_{\alpha}\right)\left(\mathrm{T}-\mathrm{T}_{\text {ref }}\right)\right]
$$

$\mathrm{CE}$ - condutividade elétrica na faixa de 15 a $35^{\circ} \mathrm{C}, \mathrm{dS} \mathrm{m}{ }^{-1}$;

$\mathrm{CE}\left(\mathrm{T}_{\text {ref }}\right)$ - condutividade elétrica à temperatura de referência, $\mathrm{dS} \mathrm{m}{ }^{-1}$;

$\alpha$ - coeficiente de temperatura, $\%{ }^{\circ} \mathrm{C}^{-1}$, e

SE $\alpha$ - desvio-padrão associado ao coeficiente $\alpha$. 


\section{RESULTADOS E DISCUSSÃO}

Os gráficos com as equações de regressão que relacionam freqüência e resistência, correspondentes ao circuito de condutividade, são apresentados nas Figuras 2 e 3 para as faixas de condutividade elétrica de 0,1 a $2,15 \mathrm{dS} \mathrm{m}^{-1}$ e 2,15 a $10,15 \mathrm{dS} \mathrm{m}^{-1}$, respectivamente. Na Figura 4, são apresentados os resultados da mesma relação, porém, para o circuito de temperatura.

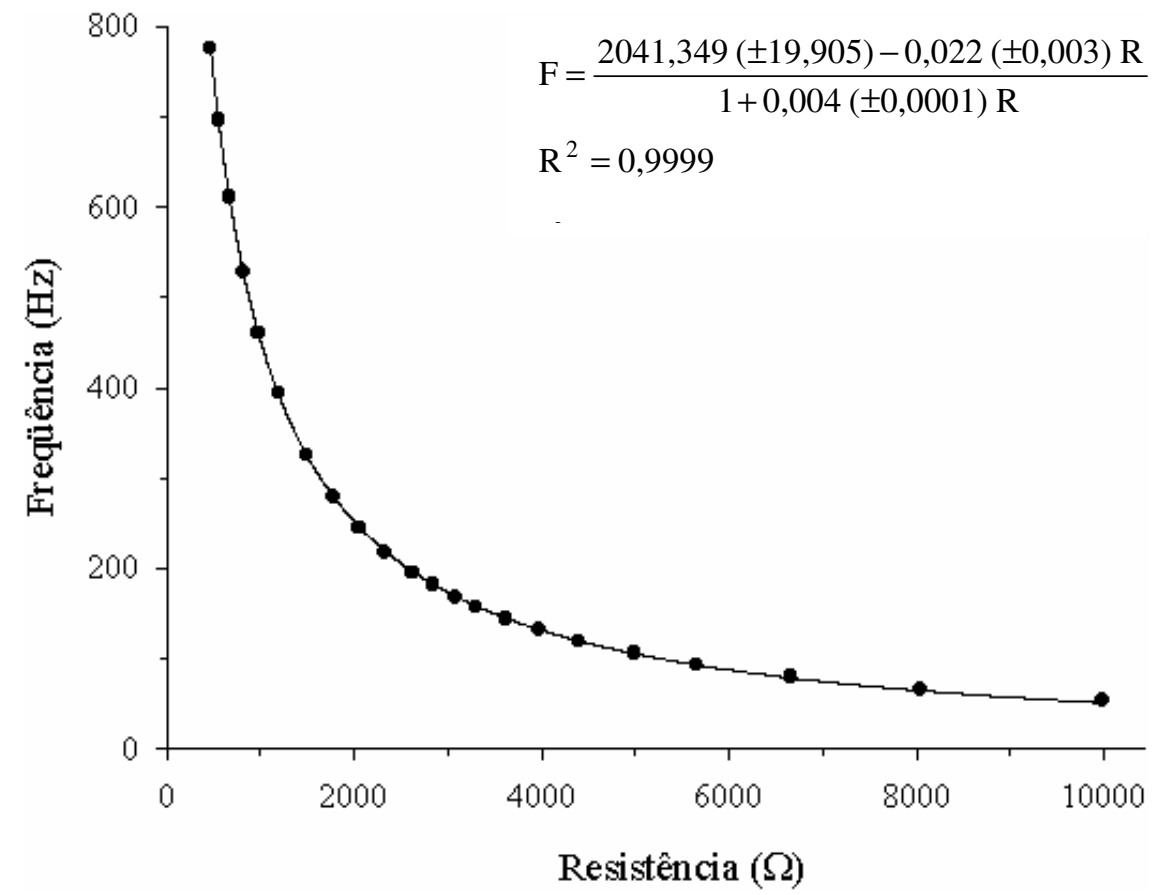

FIGURA 2. Freqüência $(F)$ de oscilação da tensão elétrica em relação à variação da resistência $(R)$, para aplicação em medições de condutividade elétrica entre 0,1 e 2,15 dS m m $^{-1}$.

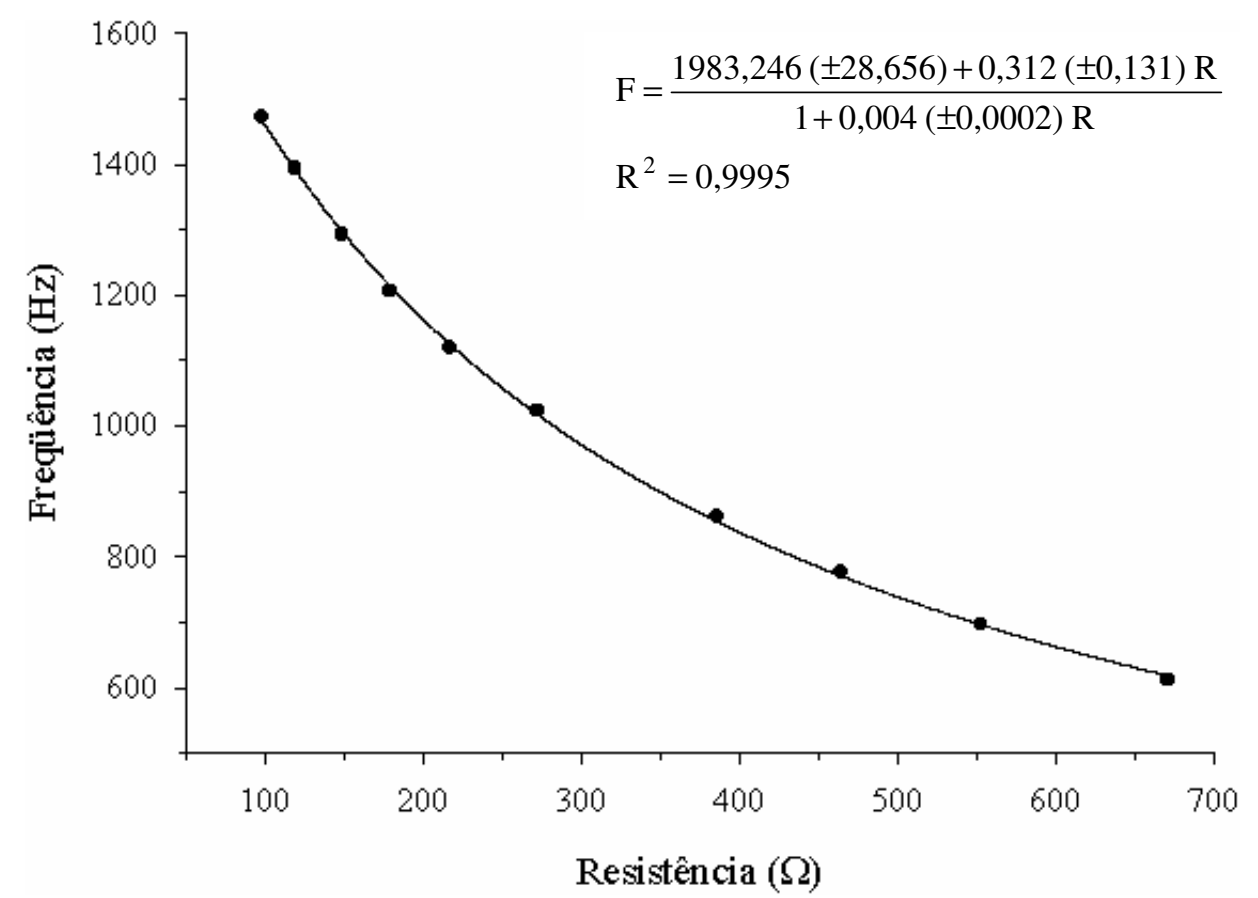

FIGURA 3. Freqüência $(F)$ de oscilação da tensão elétrica em relação à variação da resistência $(R)$, para aplicação em medições de condutividade elétrica entre 2,15 e 10,15 dS m m $^{-1}$. 


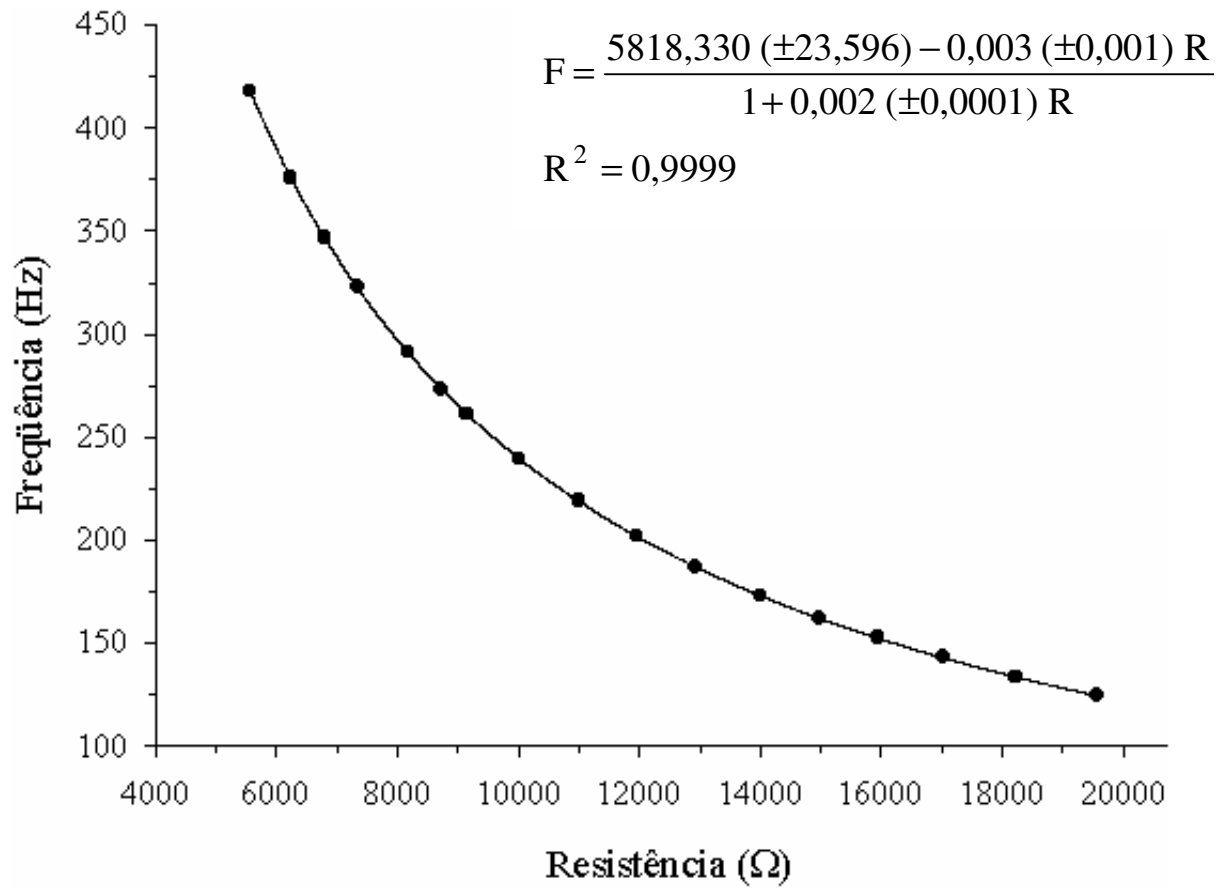

FIGURA 4. Frequiência (F) de oscilação da tensão elétrica em relação à variação da resistência $(\mathrm{R})$, para aplicação em medições de temperatura entre 283 e $313 \mathrm{~K}\left(10\right.$ e $\left.40{ }^{\circ} \mathrm{C}\right)$.

Observando-se as Figuras 2; 3 e 4, verifica-se que a frequiência decresce exponencialmente com o aumento da resistência. Nas Figuras 2 e 3, como a condutividade elétrica é inversamente proporcional à resistência, valores altos de freqüência implicam condutividades elétricas elevadas e vice-versa.

A equação e o gráfico correspondentes à regressão entre os valores de resistência e temperatura, obtidos a partir da calibração do circuito de temperatura, são apresentados na Figura 5.

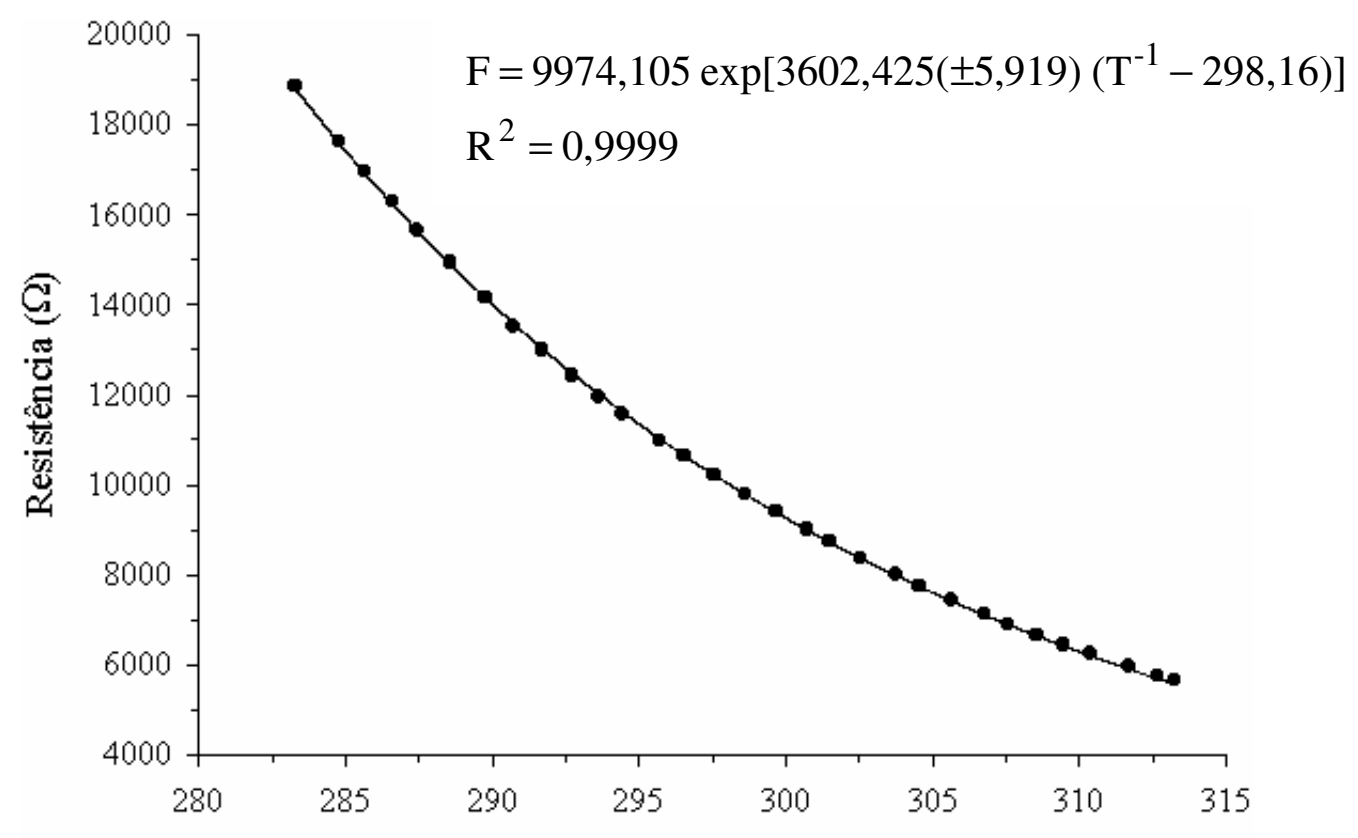

Temperatura $(\mathrm{K})$

FIGURA 5. Efeito da temperatura (T) na resistência (R), com base em medições obtidas por meio do termistor incluso na célula de condutividade elétrica. 
Conforme Figura 5, à medida que a temperatura aumenta, os valores de resistência elétrica tornam-se menores, seguindo o mesmo comportamento dos gráficos anteriores. Tal comportamento já era esperado, visto que o termistor da célula de condutividade possui coeficiente de temperatura negativo (NTC). $\mathrm{O}$ valor do parâmetro $\beta$ na equação que relaciona resistência e temperatura depende do material que constitui o termistor. Na equação apresentada na Figura 5, o valor de $\beta$, obtido a partir da regressão, está próximo do mencionado por WANG \& FELTON (1983), que é de $\approx 4000 \mathrm{~K}$ para os termistores mais comuns.

Relacionando as Figuras 2; 3; 4 e 5, é possível concluir que a condutividade elétrica aumenta com o acréscimo de temperatura. $\mathrm{O}$ gráfico apresentando os dados experimentais (pontos), juntamente com os estimados pela equação de regressão múltipla (retas), é apresentado na Figura 6.

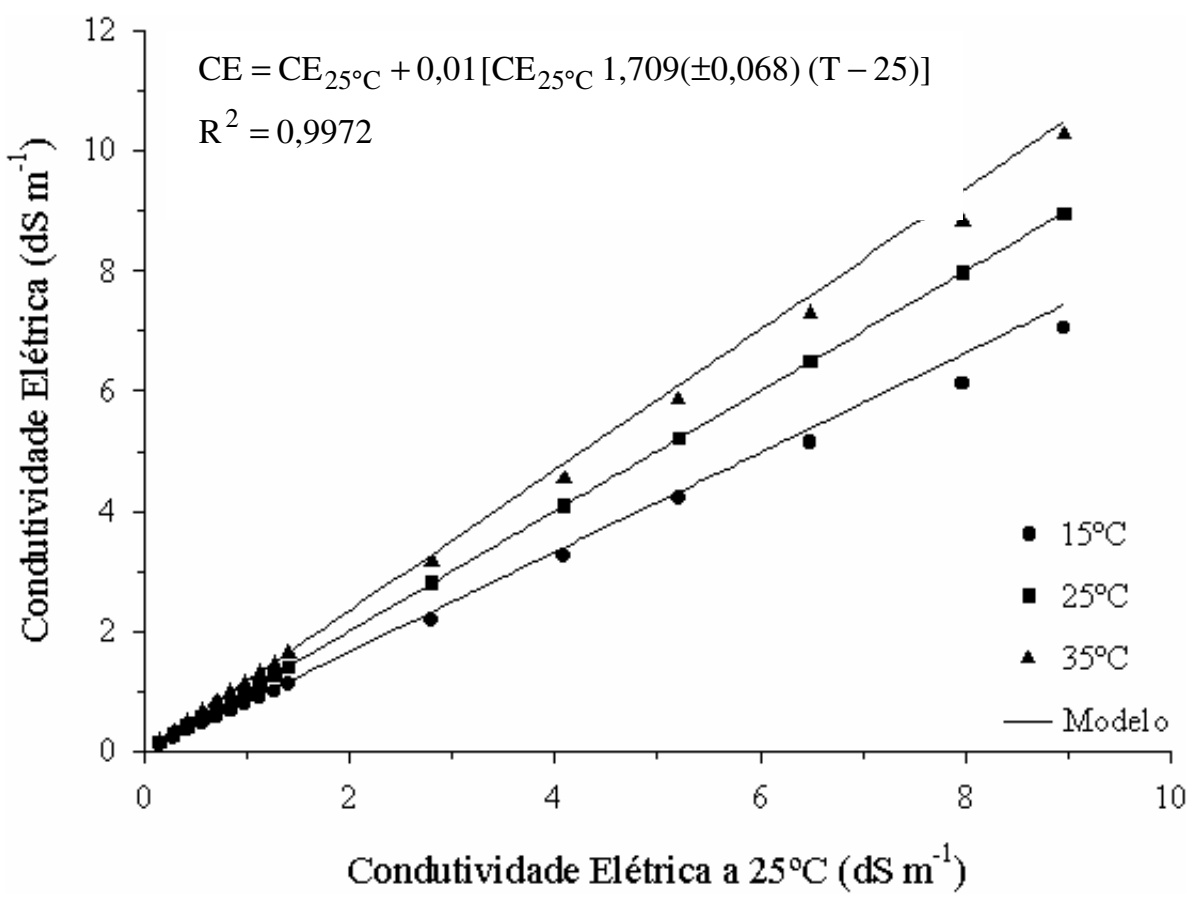

FIGURA 6. Efeito da temperatura na condutividade elétrica de soluções aquosas de cloreto de potássio, tendo como referência o valor de $25^{\circ} \mathrm{C}$.

O coeficiente de temperatura $(\alpha)$, apresentado na equação da Figura 6, aproximou-se do valor $1,9 \%{ }^{\circ} \mathrm{C}^{-1}$ citado por Wissenschaftlich Technische Werkstätten (WTW, 2002) para soluções de cloreto de potássio com concentração de $0,01 \mathrm{M}$ à temperatura de referência $\left(25^{\circ} \mathrm{C}\right)$. De acordo com ZOLNIER (2004), a maioria dos condutivímetros compensa o efeito da temperatura considerando-se a variação de $2 \%$ na condutividade elétrica para cada $1{ }^{\circ} \mathrm{C}$ na temperatura da solução.

A partir da equação de regressão múltipla, implementada no Hidro-CE, os valores de condutividade elétrica exibidos pelo programa ao usuário serão referenciados à temperatura de $25^{\circ} \mathrm{C}$.

Os coeficientes de determinação ajustados ( $\mathrm{R}^{2}$ ajustados) obtidos em todas as regressões realizadas, variaram de 0,9972 a 0,9999, demonstrando excelentes correlações entre as variáveis envolvidas nessa avaliação.

\section{CONCLUSÕES}

O circuito proposto é capaz de medir a condutividade elétrica de soluções na faixa de 0,1 a $10,15 \mathrm{dS} \mathrm{m} \mathrm{m}^{-1}$ com grande exatidão, compensando automaticamente as variações instantâneas de temperatura. $\mathrm{O}$ baixo custo dos componentes do circuito, a aquisição de dados por meio de um simples 
computador via porta paralela e a fácil adaptação a um sistema de controle tornam esse circuito ideal para usuários que desejem monitorar a condutividade elétrica de soluções nutritivas de maneira contínua, precisa e com custo reduzido. Portanto, o método proposto para a medição da condutividade elétrica tem enorme potencial para contribuir no desenvolvimento da automação de sistemas de cultivo hidropônicos e que utilizem a fertirrigação no Brasil.

\section{REFERÊNCIAS}

AXELSON, J. Parallel port complete: programming, interfacing and using the PC's parallel printer port. 1996. Disponível em: <http://www.lvr.com/parprtib.htm\#Chapter1>. Acesso em: 27 out. 2004.

DeFELICE, T.P. An introduction to meteorological instrumentation and measurement. New Jersey: Prentice Hall, 1998. 229 p.

FURLANI, P.R.; SILVEIRA, L.C.P.; BOLONHEZI, D.; FAQUIN, V. Cultivo hidropônico de plantas. Campinas: Instituto Agronômico, 1999. 52 p. (Boletim Técnico, 180)

GARTLEY, K.L. Recommended soluble salts tests. 1995. Disponível em: <http://ag.udel.edu/extension/Information/Soil_Testing/CP10-95.pdf>. Acesso em: 17 abr. 2004.

MARTINEZ, H.E.P. O uso do cultivo hidropônico de plantas em pesquisa. Viçosa - MG: Universidade Federal de Viçosa, 1999. 47 p. (Cadernos Didáticos, 1)

RADIOMETER ANALYTICAL. Conductivity - theory and practice. France, 2004. Disponível em: <http://www.radiometer-analytical.com/pdf/meterlab>. Acesso em: 7 jul. 2004.

WANG, J.Y.; FELTON, C.M.M. Instruments for physical environmental measurements. $2^{\text {nd }}$ ed. Dubuque: Kendall/Hunt Publishing Company, 1983. 317 p.

WTW. Conductivity primer. Wissenschaftlich Technische Werkstätten GmbH. Germany, 2002. Disponível em: <http://www.wtw.com/downloads>. Acesso em: 10 jul. 2004.

ZOLNIER, S. Automação de sistemas de cultivo em substrato. In: ENCONTRO NACIONAL SOBRE SUBSTRATO PARA PLANTAS, 4., 2004, Viçosa. Anais... Viçosa - MG: Universidade Federal de Viçosa, 2004. p.158-89. 